\title{
Vom Messen und Wiegen
}

\author{
E. Danieli
}

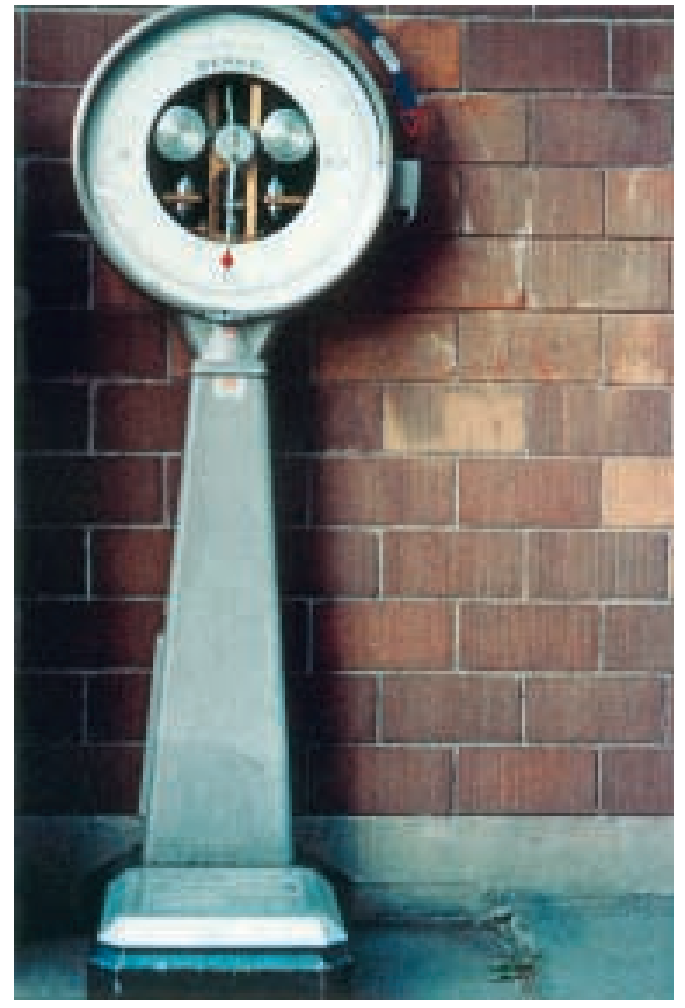

Alle Kinder, heisst es in einer Zeitungsmeldung vom 28. Mai 2004, sollen zum Fettest! «In Grossbritannien ist ein Fettest geplant. Diese Messung des eigenen Fetts müssen Kinder künftig jedes Jahr überstehen, wie ein aktueller parlamentarischer Bericht aus England vorsieht. Dadurch könne verhindert werden, dass die Kinder wegen der mit den Fettpolstern verbundenen Krankheiten früher sterben als ihre Eltern.»

Meinte nicht Hofrat Behrens: «Messen kann nie was schaden»?

Bei uns damals nicht zur Festlegung der Illumination wegen tuberkulöser Verdächtigungen, sondern aus einfacheren Gründen: Hinter der Badezimmertüre hing die Messlatte, Vaters Messstab, natürlich zur Messung unserer Körpergrösse. Jährlich zum Geburtstag gehörten genauso wie die Geschenke ritualhaft die Längenmessungen. Mit Rotstift wurde unser Wachstum, nicht ohne Stolz, festgehalten: Ein dicker Querstrich, die Initialen, das Datum.

Das Gewicht aber, nach dem Samstagabendbad monatlich kontrolliert, brauchte nicht no- tiert zu werden, diese Zahl merkten wir uns. Auf das Gewicht wurde Wert gelegt, denn ein unternormales, normales oder übernormales Gewicht widerspiegelte die entsprechende Seelenlage: $\mathrm{Zu}$ einem gesunden Geist gehört ein normales Gewicht. Lachen auf der Waage war streng verboten, und doch kam es uns vor, als schwebten wir auf dem beweglichen, mit einer Gummimatte rutschfest gemachten Teller der Personenwaage, und dass der rote Zeiger zum Adjustieren von Erwachsenenhänden je nach Lust und Laune, so schien es uns, nach oben oder nach unten gedreht wurde, erheiterte uns zusätzlich - genau wie das Schamgefühl, denn nackt wurden wir gewogen, hier galten bis zur hochbeinigen Zeit und also bis zu unserer Verweigerung keine Kleiderabzüge. Und sicher nie einen Gedanken daran, dass wir zu dick, also zu schwer für unser Alter waren: Nein, denn eher wurden wir als zu leicht befunden: Beim Arzt auf jener Waage nun Genauigkeit: Eine Säulenwaage mit verschieden grossen, walzenförmigen Laufgewichten - mit Gewichten wurde also unser Gewicht gewogen! - auf Augenhöhe, mit verchromten Zungen, die zur Deckung gebracht wurden, dann eine Zahl mit drei Stellen nach dem Komma: Ernsthafter ging es nicht. Schon möglich, dass nach dem Messen und Wägen der Appetitmangel oder gar die Blutarmut zum Thema wurde, dass vermehrter Aufenthalt im Freien, nach Möglichkeit wenig bekleidet, und gute Lüftung der Wohnung vorgeschlagen wurden, dass aber gleichzeitig vor zuviel Schleckerei - Bärendreck, Süssholz, Schleckstengel! - gewarnt wurde und dass zuletzt von Malzextrakten oder -präparaten, von Heliomalt, von Leber- oder Fischtran, von Sanasol, von der Höhensonne und vom Luft- oder Sonnenbad die Rede war.

Eine willkommene Abwechslung zur häuslichen Messerei und Wägerei stellte die grosse, rot gestrichene Waage beim Eingang zur Badeanstalt dar. Da stand die schwerfällige Zeigerpersonenwaage, die nach Einwurf von 10 oder 20 Cts. nicht nur die Schwere am Zifferblatt ablesbar machte, sondern das Gewicht auch gleich auf einem Kartonbillett ausdruckte, einer Karte, auf welcher das persönliche Gewicht schriftlich und also unleugbar festgehalten und auf deren Rückseite farbig ein Auto oder eine Lokomotive abgebildet war, eine Fotografie, die zum Sam- 
meln und Wiederwägen verleitete. Und nicht nur vor den Schwimmbädern fanden sich diese Waagen, sondern auch in Bahnhöfen neben den Schaltern: Ideale Orte, um sich vor der Reise zu messen und um sich bei entsprechend erfülltem Sollgewicht auf die Reise zu freuen, denn neben den Waagen hingen grosse Tabellen, die ermöglichten, das eigene Durchschnittsgewicht entsprechend der Körpergrösse abzulesen, allerdings galten diese Tabellen erst ab einem Alter von 10 Jahren.

Über das Untergewicht, das Normalgewicht, das Sollgewicht (Körpergrösse minus 100), über den Broca-Index (bei Männern minus 10\%, bei Frauen minus 15\%) und über das Idealgewicht wurden wir im Studium, viel später, unterrichtet. $\mathrm{Zu}$ merken hatten wir uns, dass ein zehnjähriger Knabe von 140 cm Scheitel-Fuss-Länge 30 Kilogramm wiegt, dass nun aber besser von
Standhöhe statt von Grösse zu sprechen ist, dass es die Perzentilenkurven wie sonst nichts zu beherrschen gilt und dass auch die Ober- oder Sitzhöhe, die Stammlänge, die Spannweite, die Ober- und Unterlänge zu kontrollieren ist, denn die Pubertät sei das Storchenzeitalter, die Beine seien dann relativ am längsten, also gelinge es, mit derartigen Messungen am leichtesten diese Diagnose zu stellen, und dann, noch später, war, im Zusammenhang mit der nun grassierenden Fettsucht, die Rede vom Falstafftyp, von der Reithosenfettverteilung, von Apfel- und Birnenformen, von gynoid und von androgyn, und noch viel später, eleganter und bedeutender und alles umfassend, alles integrierend und endlich alles beinhaltend: der Body-Mass-Index und die Waist-to-hip-Ratio.

Hatte Hofrat Behrens doch recht: «Messen kann nie was schaden»!

\title{
Nachtmilch
}

\author{
E. Danieli
}

Korrespondenz:

Dr. med. Enrico Danieli Seefeldstrasse 128 CH-8008 Zürich
«[...] auf dem weissen Tischchen daneben brannte die elektrische Lampe und stand neben einem Stapel Bücher ein Glas fetter Milch, die Abendmilch, die allen Bewohnern des «Berghofs» noch um neun Uhr aufs Zimmer gebracht wurde [...].»

«Still, still, still, wenn das Kindlein schlafen will.» Brustmilchernährte Kinder sollen nicht nur während der Stillzeit, sondern auch noch lange Zeit danach besser schlafen als Kinder, die nie gestillt wurden. Liegt es vor allem an der $\mathrm{Zu}$ wendung? Oder an der spezielleren Milch? War es die Angst vor der Verlassenheit, der Dunkelheit, die Angst vor den Träumen, der nächtlichen Heimsuchung, die mich nicht schlafen liess nicht schlafen lässt? Später war von mangelndem Urvertrauen die Rede, von fehlender Tagesmüdigkeit, auch einmal von einem unguten Gewissen, das ein schlechtes Ruhekissen sei. Oder, noch später, soll es die Angst vor der letzten Nacht, vor dem ewigen Schlaf sein, die zur Schlaflosigkeit zwinge. Aber sind wir denn nicht, wenn wir schlafen, weit fort und wehrlos, sind wir nicht, wenn wir schlafen, immer auch allein? Denn Schlaf ist unteilbar. Früh schon kam mir der Gedanke, im Schlaf, vielleicht nur für kurze Zeit, tot zu sein: Obwohl ich noch da war, war ich abwesend. War das denn mit dem Tod nicht ganz ähnlich? Aber mit diesem Schattenreich wollte ich nichts zu tun haben.

Wohliges Bett und Schlafzimmerdunkelheit sollen helfen, ruhig zu schlafen. Aber schon früh beginnt das Martyrium unnützer Besänftigungsmethoden: kalt oder warm duschen, ein warmes Sitzbad, Bienenhonig als Apitherapeutikum, als Konstitutionsmittel, als Lebenselixier, wie es $\mathrm{Pa}$ racelsus versteht, denn Honig soll kosmische Ordnung, Licht in den Leib bringen und auch noch nach dem Tod als Elixier wirken, dann Melissengeist, Holundersaft, Hopfen, Essigumschläge, Bauchwickel, Stirnkompressen, Bettflaschen, Lutschtücher. Und immer die Abendmilch: rohe, kalte Milch, warme Milch, Honigmilch oder nur Milchzucker.

Nicht überrascht also bin ich vom neuartigen Angebot der Nacht- oder Schlafmilch für Schlaflose: Die Besonderheit dieser Milch ist deren Gehalt am Schlafhormon Melatonin. Werden die Kühe am frühen Morgen, nach acht Stunden in völliger Dunkelheit ebenfalls im Dunkeln der Stall mit Brettern verdunkelt, die Melker verrichten ihre Arbeit im Schein blauer Partylampen oder mit Taschenlampen -, gemolken, 
enthält die Milch das Hormon in mindestens fünffacher Konzentration. Der Erfolg vergleichbarer Produkte gegen Schlafstörungen in Finnland stimme zuversichtlich. Dunkelheit, Nacht, geschlossene Augen, wie wir wissen, stimulieren Synthese - via Lichtinformationen der Retina und Freisetzung von Melatonin in der Epiphyse. Melatonin hat nach Ansicht von Fachleuten eindeutig schlaffördernde Wirkung, vor allem, wird berichtet, bei älteren Leuten und bei Personen mit verändertem Tag-und-Nacht-Rhythmus. Melatonin, von vielen Millionen Menschen ohne jede ärztliche Verschreibung eingenommen: ein unkontrolliertes Massenexperiment also, das wohl grundsätzlich bestätigt, dass Melatonin, wann und wieviel auch immer, wirkt. Die üblicherweise verwendeten Dosen (1-5 mg) scheinen auch keine nennenswerten Nebenwirkungen zu haben (Hang-over, Hypothermie, Konzentrationsstörungen). Für Kinder allerdings soll Melatonin ungeeignet sein, hängt doch möglicherweise die sexuelle Reifung mit der allmählichen Abnahme der Melatoninsekretion während der Kindheit zusammen.

Vielleicht deswegen folgten trotz aller Bemühungen damals Tränen, Reizbarkeit. Von unruhiger Ruhe, störender Ruhelosigkeit wurde geredet, und selbst die sogenannten Schlafpräparationen, das Einschlafritual, blieben erfolglos: $\mathrm{Zu}$ den Wickeln und zu den Gutenachtgeschichten, zum Licht-brennen-Lassen und zur spaltbreit offenen Zimmertüre kamen nach dem Nachtgebet, nach dem Amen und nach dem Gutenachtkuss noch die Sprüche und die Abzählverse hinzu: «Stiefel muss sterben, wenn das der Absatz wüsst', dass Stiefel sterben müsst'...», «Es war einmal ein Mann, der hatte einen Schwamm, der Schwamm war ihm zu nass, da ging er auf die Gass'...», Zahlreihen vor und zurück, Schäfchen- und Sternenzählen. Wann genau, fragte ich sinnloserweise, habe ich denn bloss nur meine Augen zu schliessen? Doch umsonst. Obwohl vielleicht dies der wesentlichste Punkt gewesen wäre. Nie ging ich wie Proust früh zu Bett, denn vor dem Zufallen der Augen fürchtete ich mich, zum Lidschluss, ja nicht einmal zu einem Halb- oder Wachschlaf kam oder kommt es nicht, aber auch habe ich mich nie gesträubt gegen das Schlafengehen. Wozu auch? Ich sei, heisst es nun, gar nicht willfährig dem Schlaf gegenüber. Doch einen Vorteil haben wir Schlaflosen: Uns bleibt das trübe, unglückliche Erwachen erspart, das schwere Aufschlagen der Lider in dunkler Frühe. Denn ohne Erwachen gibt es vielleicht auch kein Vergehen, also gar keine Vergänglichkeit. Vom Schattenreich des Zauberbergs also sind wir weit entfernt.

Doch jetzt, mit der Schlafmilch, wer weiss, wird alles ganz anders: Vielleicht schlafe ich schon bald - und dann ganz sicher mit weit offenen Augen. 